\title{
Axial Position Estimation of Conical Shaped Motor for Green Taxiing Application
}

\author{
S. Roggia, M. Gaelea, C. Gerada \\ Faculty of Engineering, \\ University of Nottingham, \\ Nottingham, UK \\ Email:sara.roggia@nottingham.ac.uk
}

\author{
F. Cupertino \\ Department of Electrical and Information Engineering,, \\ Politecnico Di Bari, \\ Bari, IT
}

\begin{abstract}
This paper considers the possibility of adopting a conical shaped motor for Green Taxiing (GT) application. This topology of motor has been selected in order to obviate the presence of external declutching system (i.e. mechanical or electromagnetic clutch) interposed between the electric actuator and the wheel. An axial force contributes to move the rotor inside-out of the stator (principle of sliding-rotor). The axial movement of the rotor can be monitored acting on the magnetizing current. The axial sensor-less position estimation method described hereafter envisages the possibility of evaluating the axial position of the rotor during the engaging and disengaging movement from the wheel. The axial position calculation is dependent on the inductance of the motor. An "online" computation of the position has been implemented through the use of high-frequency injection signals.
\end{abstract}

Keywords-conic motor; sliding rotor; high voltage signal injection; green taxiing

\section{INTRODUCTION}

The Green Taxiing ${ }^{\mathrm{TM}}$ initiative (GT) is a scheme launched in 2008 by Safran - Messier Bugatti Dowty [1], with the main aim of manoeuvring aircraft on the ground without having to utilise the main engines or the traditional airport tow trucks. This is achieved by implementing a controlled electric motor actuator in the main landing gear (LG) of the aircraft.

Focal requirement for such an application is to achieve the maximum torque rate minimizing volume taken by the electric motor inside the landing gear. Though, the best electrical machine configuration in terms of torque density can be achieved using special design techniques [2], other issues, concerning the whole system configuration, should be considered.

The electric actuator can be designed to be directly coupled and always connected to the wheel [3], spinning together at any velocity. This topology of actuator is called Direct Drive (DD) Wheel Actuator, where the adjective direct underlines that no mechanical device is interposed between the electric motor and the wheel. With a DD motor, high figures of reliability can be achieved [4]. Additional mechanical components, in fact, increase the risk of jams whilst increasing the overall weight of the system. However, adopting this kind of configuration, excellent fault-tolerance properties are required for the motor. It must be able to withstand and sustain demanding conditions when the aircraft is performing take-off or landing. The risk of excessive voltage levels on the motor terminals, due to high speeds achieved by the wheel during the take-off and landing phases, is relevant side effect of such a kind of motor implementation. Since safety and reliability are key words in the aerospace field, solutions to actively decouple the actuator from the wheel would be highly recommended.

This research work considers the possibility of adopting a conical shaped motor to obviate the presence of external declutching system (i.e. mechanical or electromagnetic clutch) whilst having an active engagement/disengagement of the actuator during take-off and landing. The concept has been proven on an experimental test-rig. An axial sensor-less position estimation method has been built on an "off-the-shelf" asynchronous conical motor with the aim of evaluated the position of the motor in the wheel before performing take-off and landing.

\section{CONICAl Shaped Motor Principles AND THEORY}

\section{A. Forces Acting On Conical Motor}

A conical motor is an electrical machine in which both stator and rotor have conical shape, determined by the angle calculated at vertex of the cone (cone angle) [6]. Due to its particular shape, forces acting on the motor contribute to move the rotor inside-out of the stator (principle of sliding- rotor). The movement of the rotor will cause air-gap variation that will correspond to a specific electrical and magnetic configuration of the machine, related to the rotor position inside the stator. As in cylindrical electrical machines, three different kinds of forces act on the rotor: lateral or radial, tangential and axial force. Particularly, with respect to the lateral surface, the total force can be divided into tangential and normal components [5], [6].

Tangential forces, via Lorentz Forces, acting on rotor and stator, are responsible for producing the required torque. Radial and axial forces are generated from normal component of the total force oriented normally to the lateral surface. In a magnetically balanced motor, total effect of radial forces tends to be null due to the fact that the vector sum of radial stresses is almost equal to zero. Axial forces will depend on the shape of the motor. This contribute is negligible in cylindrical motors. However, in a conical shaped motor, the generated axial force 
has two contributions (Fig. 1): the amount of force due to the difference between forces acting normally to surfaces $S_{1}$ and $S_{3}$ and the axial component of force acting normally to conical lateral surface $\mathrm{S}_{2}$.

The total force on $\mathrm{S}_{2}$ is the sum of two components: the radial component $F_{\mathrm{r}}$ and the axial component $\mathrm{F}_{\mathrm{z}}$. The presence of this axial component results in an axial movement of the rotor that if controlled appropriately can be used to provide an engagement/disengagement of the motor from the aircraft wheel.

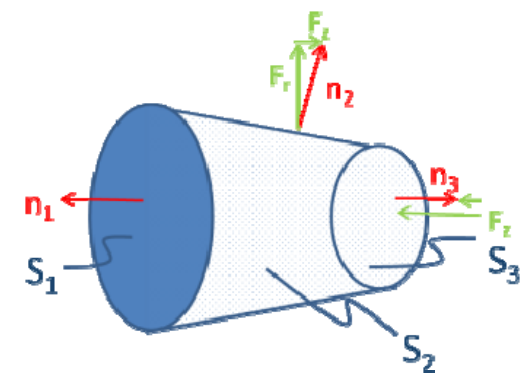

Fig. 1 Forces Acting on Conical Motors

\section{B. Axial Position Estimation Method}

In the proposed method, the conical asynchronous motor has been modelled by means of space vectors as a common asynchronous one. Voltage equations can be expressed both in time-dependent stationary reference frame $\alpha \beta$ (1) and timeindependent synchronous reference frame $d q(2)$.

$$
\begin{gathered}
\mathbf{u}_{s}^{\alpha \beta}=R_{s} \mathbf{i}_{s}^{\alpha \beta}+\sigma \mathbf{L}_{s} \frac{d \mathbf{i}_{s}^{\alpha \beta}}{d t}+\frac{L_{M}}{L_{R}} \frac{d \lambda_{R}}{d t} \\
\mathbf{u}_{s}^{d q}=R_{s} i_{s}^{d q}+\sigma \mathbf{L}_{s} \frac{d \mathbf{i}_{s}^{d q}}{d t}+\frac{L_{M}}{L_{R}} \frac{d \boldsymbol{\lambda}_{R}}{d t}+j \omega_{\varphi} \sigma \mathbf{L}_{s}^{d q}+j \omega_{R} \frac{L_{M}}{L_{R}} \lambda_{R}
\end{gathered}
$$

subscripts $\mathrm{S}$ and $\mathrm{R}$ refer respectively to stator and rotor quantities, whereas $L_{M}$ is the magnetizing inductance, $\sigma$ is the leakage factor calculated as $\frac{1-L_{M}{ }^{2}}{L_{R} L_{S}}, \sigma L_{s}$ is the stator transient inductance and $\omega_{\varphi}$ is the angular speed of rotor flux space vector $\left(\lambda_{R}\right)$.

The intent is to realise a sensor-less technique for monitoring the rotor, namely its switching movement from completely disengaged position to fully engaged position simply acting on the magnetizing current. The magnetizing current, in fact, is the main responsible of the motor movement. It produces the necessary magnetic force, responsible for the attraction between the rotor and the stator. Thus, estimation strategy exploits the variation in magnetic features of the actuator when the rotor is moving inside-out of the stator.

From disengaged position to engaged position, due to reduction of the air-gap, the flux-current characteristic of the motor is translating from the right to the left in the flux-current plane (Fig. 2). Thus, inductance of the motor increases passing from one position to another, considering the same amount of magnetizing current.

For the evaluation of the position, High Frequency (HF) Injection Technique has been adopted. This methodology takes advantage of interaction between the carrier signal excitation voltage and the stator transient inductance to estimate the machine parameters [7]. In fact, saturation effects induced in the machine are capable to spatially modulate the quantity $\sigma \mathbf{L}_{s}$.

With sufficiently high frequency, the effects of the injection can be considered independent of behaviour of the motor at the fundamental frequency. The model of the motor at high frequencies can be obtained neglecting voltage drop on the resistance, as well as the other contributions [9] [10]; thus, equation (1) becomes:

$$
\mathbf{u}_{I n j}^{\alpha \beta} \cong j \omega_{I n j} \sigma \mathbf{L}_{s}^{\alpha \beta} \mathbf{i}_{s}^{\alpha \beta}
$$

where $\mathbf{u}_{I n j}^{\alpha \beta}$ is the injected signal rotating at $\omega_{I n j} \mathrm{rad} / \mathrm{s}$.

The stator transient inductance, containing saturationinduced saliency, in the synchronous reference frame can be represented as follow:

$$
\sigma \mathbf{L}_{s}^{d q}=\left[\begin{array}{cc}
\sigma L_{d s} & 0 \\
0 & \sigma L_{q s}
\end{array}\right]
$$

In the stationary (stator) reference frame, the stator transient inductance can be expressed as sum of two quantities, the average stator inductance $\sigma \mathbf{L}_{d s}$ and the differential stator inductance $\Delta \sigma \mathbf{L}_{d s}$ in the form [11]

$$
\sigma \mathbf{L}_{s}^{\alpha \beta}=\left[\begin{array}{cc}
\sigma L_{s}+\Delta \sigma L_{s} \cos \left(2 \vartheta_{e}\right) & -\Delta \sigma L_{s} \sin \left(2 \vartheta_{\mathrm{e}}\right) \\
-\Delta \sigma L_{s} \sin \left(2 \vartheta_{\mathrm{e}}\right) & \sigma L_{s}-\Delta \sigma L_{s} \sin \left(2 \vartheta_{\mathrm{e}}\right)
\end{array}\right]
$$

where $\vartheta_{e}$ is the angle of rotating saliency and $\sigma L_{s}=\frac{\sigma L_{q s}+\sigma L_{d s}}{2}, \Delta \sigma L_{s}=\frac{\sigma L_{q s}-\sigma L_{d s}}{2}$.

The average stator inductance and the differential stator inductance can be represented respectively as the angular coefficient of the linearized flux-current characteristic and the tangent to the real curve, as indicated in Fig. 2.

When high frequency carrier signal is superimposed on the fundamental excitation, a carrier signal current with same frequency of the injected voltage $\left(\omega_{I n j}\right)$ will be induced in the stator. The rotating high frequency voltage is generated by injecting in the stationary frame $\alpha \beta$, on both axis, continuous signals with same amplitude $\left(U_{I n j}\right)$ and frequency much higher than the fundamental $\left(\omega_{I n j} \gg \omega_{R}\right)$. The injected signal describes a circumference in the stationary reference frame (with radius equal to $U_{I n j}$ and centre in the origin of the reference frame). It is implemented in the following form: 


$$
\mathbf{u}_{I n j}^{\alpha \beta}=\left[\begin{array}{l}
u_{\alpha I n j} \\
u_{\beta I n j}
\end{array}\right]=U_{I n j}\left[\begin{array}{c}
\cos \omega_{I n j} t \\
\sin \omega_{I n j} t
\end{array}\right]=U_{I n j} e^{j \omega_{I n j} t}
$$

The high frequency current signal consists of positive and negative sequence components, formulated in the stationary reference frame in the form

$$
\mathbf{i}_{s}^{\alpha \beta}=\left[\begin{array}{l}
i_{s \alpha} \\
i_{s \beta}
\end{array}\right]=\left[\begin{array}{l}
I_{p} \cos \omega_{l l j} t+I_{n} \cos \left(2 \vartheta_{e}-\omega_{l n j} t\right) \\
I_{p} \sin \omega_{l n j} t+I_{n} \sin \left(2 \vartheta_{e}-\omega_{l n j} t\right)
\end{array}\right]=I_{p} e^{j \omega_{n n t} t}+I_{n} e^{j\left(2 \vartheta_{e}-\omega_{l n j} t\right)}
$$

where the positive and negative amplitudes of the current, respectively $I_{p}$ and $I_{n}$ have the following expression

$$
I_{p}=\frac{U_{l n j}}{\omega_{l n j}} \frac{\sigma L_{s}}{\sigma L_{s}^{2}-\Delta \sigma L_{s}^{2}} ; \quad I_{n}=\frac{U_{l n j}}{\omega_{l n j}} \frac{\Delta \sigma L_{s}}{\sigma L_{s}^{2}-\Delta \sigma L_{s}^{2}}
$$

The positive sequence is proportional to the average stator transient inductance and does not contain any information about saliency. The negative sequence carrier signal current is proportional to the differential stator inductance and arise from different topologies of saliency, as summarized in [8], [12].

In this study, the estimation of axial rotor position is based on the analyses on the negative sequence carrier signal. Containing the differential stator transient inductance information, this sequence is subject to a sensible variation in amplitude between disengaged and engaged phase. This concept emerges clearly from Fig. 2, where the tangent to the flux-current characteristic assumes appreciable different inclinations between disengage and engaged cases for a rated magnetizing current. On the contrary, considering the same working point, the average stator transient inductance values of the two curves can result in being very similar.

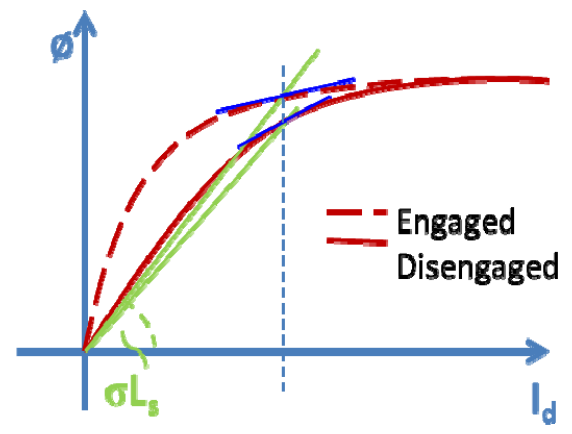

Fig. 2 Average and Differential Stator Transient Inductance Representation in Flux-Magnetizing Current Reference Frame

Whilst the high-frequency negative sequence component of the current response will be used for the axial position computation, the fundamental frequency counterpart will operate as feedback signal for the current control loop.

For this purpose, the whole current signal needs to be processed and demodulated in order to isolate two different frequency levels of the current. This operation is implemented in the Filtering and Demodulation Block of Fig. 3. The feedback current of the current control loop component is obtained through the use of a Notch Filter, excluding the injection frequency component of the current. On the other side, the demodulation of the negative sequence carrier current can be achieved shifting negative-sequence to $\mathrm{dc}$ and attenuating the fundamental-frequency current component through the use of a Lowpass Filter. Current carrier signal in the negative-synchronous frame assumes the form

$$
\mathbf{i}_{s}^{n}=\mathbf{i}_{s}^{\alpha \beta} e^{j \omega_{h y j} t}
$$

Filter design as well as other important aspects of the control system (i.e. the selection of the injection frequency) will be described in the next section as strictly related to the motor under test.

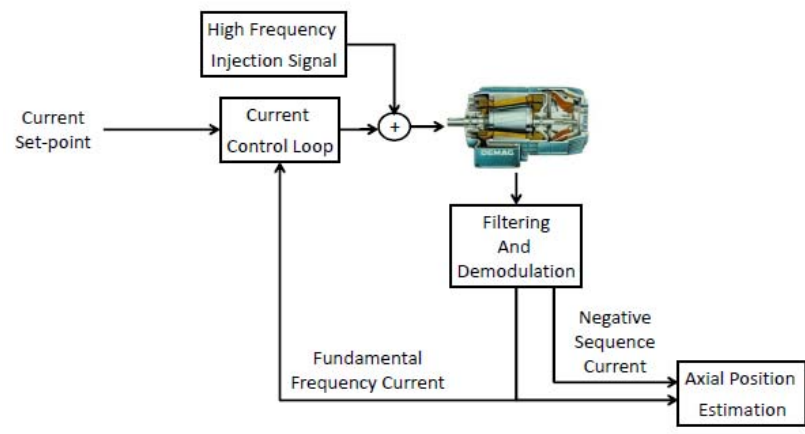

Fig. 3 Average and Differential Stator Transient Inductance Representation in Flux-Magnetizing Current Reference Frame

\section{EXPERIMENTAL TESTS}

The axial position estimation method has been tested on "off-the shelf" conical motor (Fig. 4) with rated speed of 500 rpm, $14 \mathrm{Nm}$ torque at $50 \mathrm{~Hz}$ frequency, $4.6 \mathrm{~A}$ current for wye connected $400 \mathrm{~V}$ voltage supply. The motor is provided with a brake system, composed by a disk and a spring. The rotor is always disengaged from stator until the motor will develop an axial force able to overcome the spring thrust. Brake spring force is $180 \mathrm{~N}$ and clearance from completely disengaged to completely engaged position is $4 \mathrm{~mm}$.

The real-time control of the motor has been realized through a dSPACE ${ }^{\circledR}$ Digital Signal Processing (DSP) board connected to a PC. The monitoring is performed using dSPACE software graphical user interface (GUI). The control algorithm has been built in Matlab/Simulink environment and then converted and loaded onto the dSPACE DSP. Three A/D converters were used to transduce the feedback current signal and the DC link voltage measurements. Converter of $750 \mathrm{~W}$ rated power has been employed to feed the motor, equipped with three phase IGBT inverter. IGBTs features are: 4 Arms rated current, $400 \mathrm{DC}$ V rated voltage and $24 \mathrm{kHz}$ switching frequency.

In order to estimate the rate of change of the negativesequence of the carrier current signal at different positions, an experiment has been set by using a gradual increasing sequence of current steps. The magnetizing current sequence stops until the engagement is reached. Starting from this point, a 
decreasing sequence in a symmetrical way is performed for returning to disengaged position (Fig. 7).

Using the same sequence, several tests were performed on a range of frequencies from 500 to $1000 \mathrm{~Hz}$ and different amplitudes of the injected signal. Better results were obtained by means of a $500 \mathrm{~Hz}$ injected voltage, resulting in producing appreciable negative-sequence current amplitude, two times greater than the one produced by using $1000 \mathrm{~Hz}$ injected voltage. Fig. 6 compares the trend of $I_{n}$ amplitude (amplitude of the negative-sequence carrier current signal at $500 \mathrm{~Hz}$ and $1000 \mathrm{~Hz}$ ) for different levels of $I_{s d}$ current when rotor is completely disengaged and fully engaged.

The axial position estimation algorithm is able to associate the level of negative sequence current to the position of the rotor, looking at magnetizing current level and Lowpass Filter output. In order to train the algorithm, several tests have been carried out, locking the rotor at different axial position by mean of an "end-stop" plate (Fig. 5) and varying the magnetizing current. Data, as reported in TABLE I. , were recorder and analysed in order to build interpolating functions to be integrated in the estimation model. Two topologies of functions were built and combined together: a) functions reproducing the behaviour on the negative-sequence current for each level of magnetizing current when the rotor axial position varies from 0 to $4 \mathrm{~mm}$ (Fig. 8); b) functions that track the behaviour of the negative-sequence current for each axial position when the level of magnetizing current increase from $1.5 \mathrm{~A}$ to $4.8 \mathrm{~A}$ at which engagement happens (Fig. 9). All these functions allow having a 3D meshed surface, similar to the one in Fig. 10. The axial position observer, receiving $I_{s d}$ and $I_{n}$ as inputs, will search through the 3D surface and return the appropriate axial position as output. In order prevent from any further extrapolation once the maximum axial position is reached, a saturation block is added at the output of the estimation block.

For proving that the negative counterpart of the carrier current signal is the best signal to be associated with axial position variation, spectral analyses have been performed at any level of magnetizing current. Amplitudes of most evident harmonics have been recorded and compared during the engaging and disengaging phases. As stated in the theory, in correspondence of frequency of negative-sequence, the maximum rate of variation has been documented. Particularly, a difference up to $20 \mathrm{~dB}$ in magnitude has been reported when current is lowered from 4.8 to $2 \mathrm{~A}$ and a voltage signal of 500 $\mathrm{Hz}$ is injected (see Fig. 11).

The reference current is imposed by the current control loop. The current control feedback signals is the output of second order Notch Filter centered on the injected frequency, whereas the analysed negative sequence current is the output current of the low-pass filter. The input current of the low pass filter is the current signal translated in the negative-sequence reference frame; therefore, the signal to be attenuated is in correspondence of the injected frequency. Magnitude response and attenuation of these two filters, when a $500 \mathrm{~Hz}$ voltage signal is injected, are displayed in Fig. 12 and in Fig. 13. Filters were designed by means of traditional methods (i.e. Butterworth) [13], minimizing time response delay.
Negative-sequence current variations happen, as obvious, consistently with steps of reference current; however an additional increment should be noticed during the last highest step of Fig. 7. The magnetizing current value is equal to $4.8 \mathrm{~A}$, enough to generate the axial force that will move the rotor inside the stator. In correspondence of $7.1 \mathrm{~s}$ engagement happens and the negative-sequence current amplitude increases further (Fig. 14) even though the magnetizing current remains still the same (Fig. 7). The output of the axial position estimation algorithm, in Fig. 15, is able to track changes in negative-sequence of the carrier current signal also during this transitional phase. As noticeable in Fig. 14 and Fig. 15, negative-sequence current and axial position show really similar behaviours, as the axial position computational system has been optimized to be synchronized with changes in Lowpass filter current output.

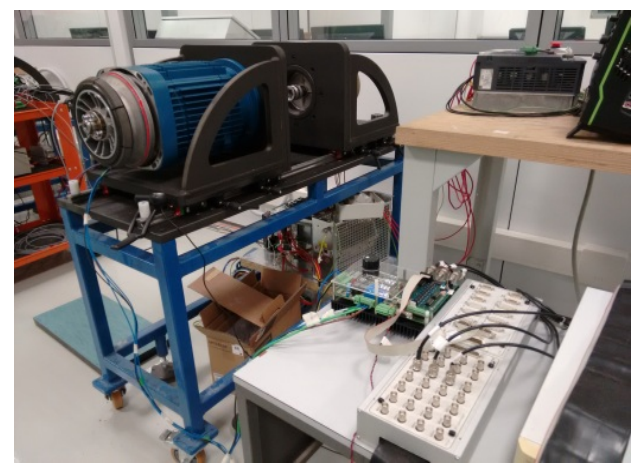

Fig. 4 Test-rig Set-up

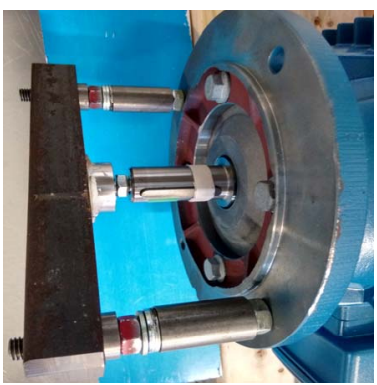

a) End-stop plate allows $0 \mathrm{~mm}$ axial movement

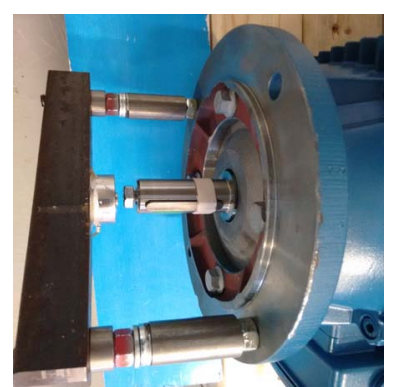

b)End-stop plate allows $4 \mathrm{~mm}$ axial movement
Fig. 5 End-Stop Configuration

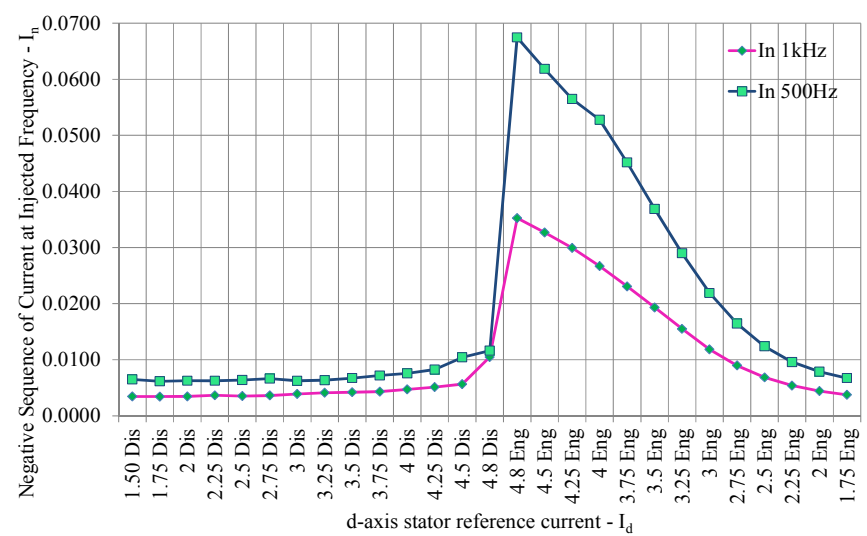

Fig. 6 Negative Sequence Current Comparison with $500 \mathrm{~Hz}$ and $1 \mathrm{kHz}$ Injected Voltage 


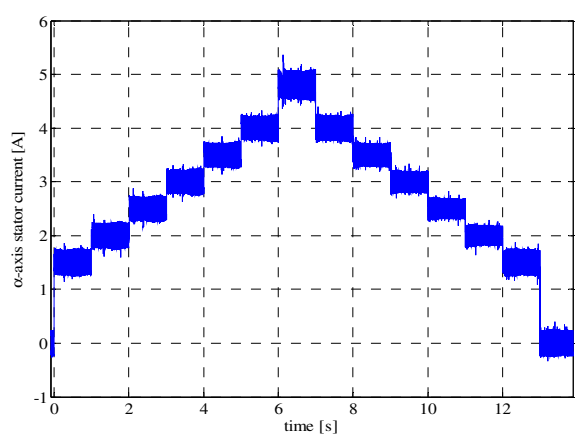

Fig. 7 Magnetizing Current Sequence

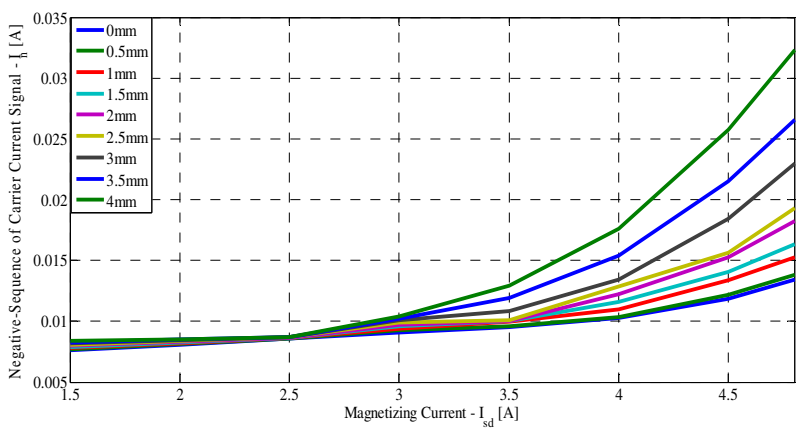

Fig. 8 Negative-sequence current for each level of magnetizing current varying rotor axial position

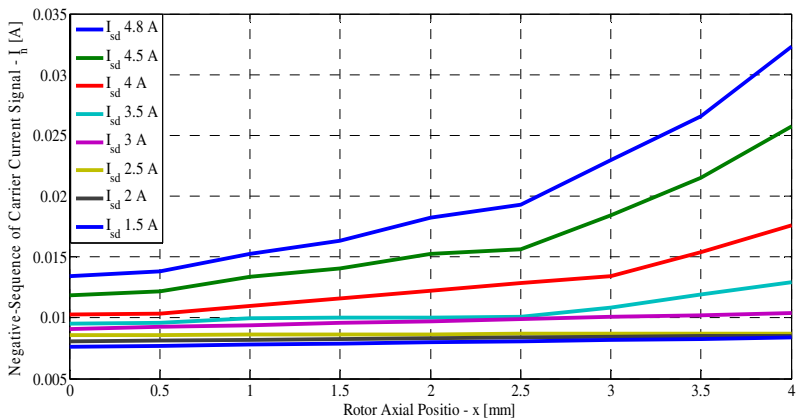

Fig. 9 Negative-sequence current for rotor axial position varying the level of magnetizing current

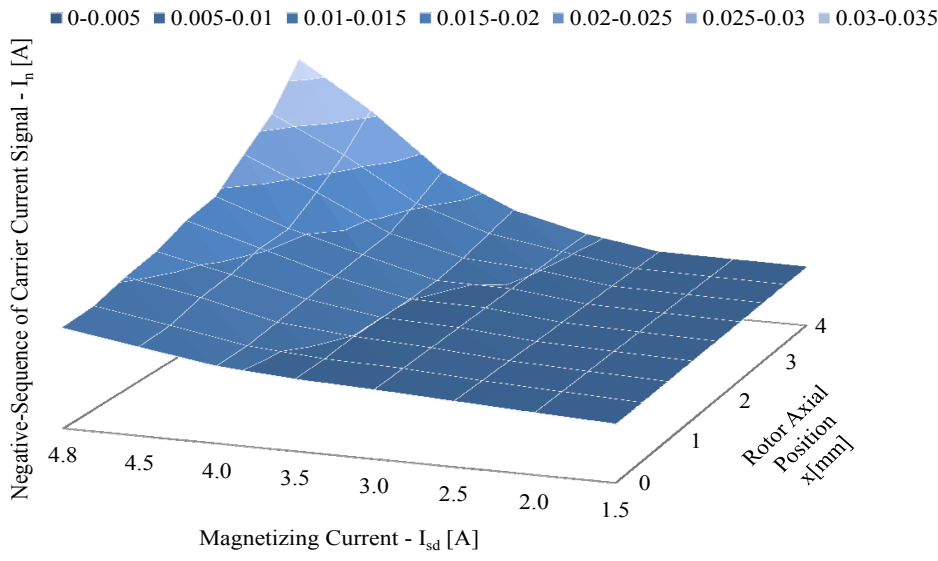

Fig. 10 3D Searching Meshed Surface for Axial Observer

\section{CONCLUSIONS}

The paper shows innovative application of a conical shaped motor in the aerospace field. The motor could represent a solution for avoiding clutch mechanism in GT system. Through the realization of an axial position observer, it is possible to evaluate the position of the actuator within the wheel. A self-commissioning scheme for the axial position estimation has been set up through the injection of high frequency voltage signal. Due to the novelty of the problem, no terms of comparison were found in the actual state-of-the-art. However, the most convenient way to estimate the axial position was found to be the tracking of saliency by means of the negative sequence of the carrier current signal. As shown, this sequence appears to be very sensible to changes of main flux passing from disengaged to engaged position. A sensorless axial position estimation will allow evaluating the rotor movement without any extra sensor, in order to safely engaging and disengaging the landing gear.

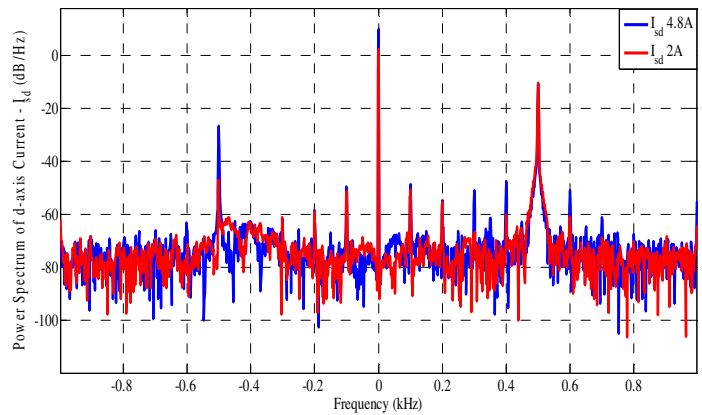

Fig. 11 Power Spectrum of d-axis current with $500 \mathrm{~Hz}$ Injected Voltage Signal

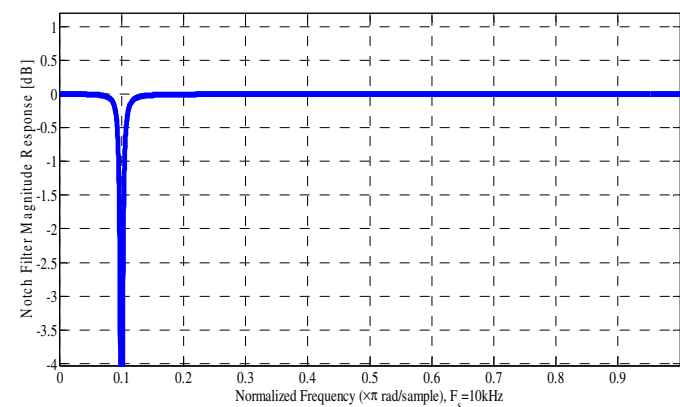

Fig. 12 Notch Filter for $500 \mathrm{~Hz}$ Injected Signal

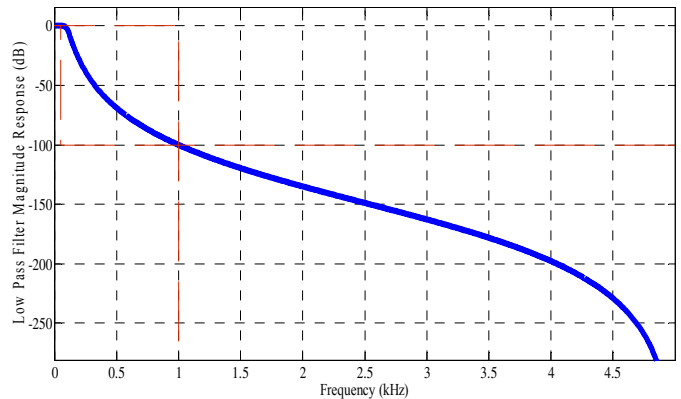

Fig. 13 Lowpass Filter Magnitude Response 


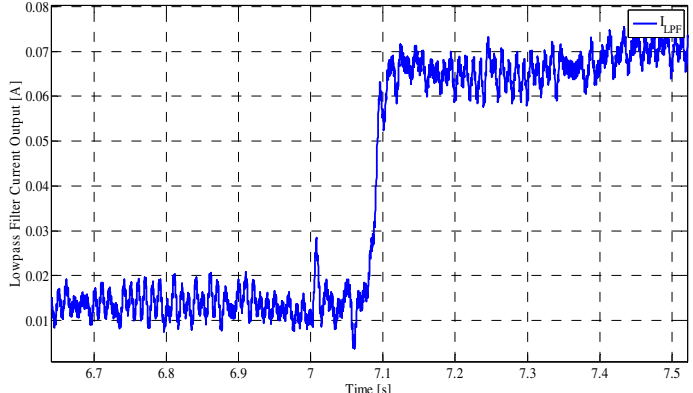

Fig. 14 Lowpass Filter Current Output during Engagement

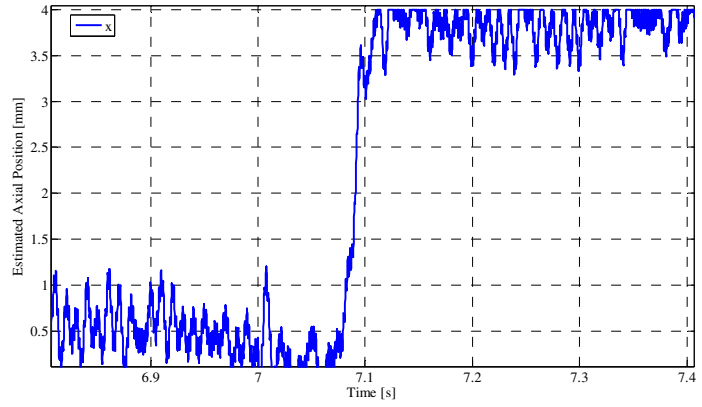

Fig. 15 Axial Positon Estimation Algorithm Output during Engagement

TABLE I. MeAsured Negative-Sequence of CARrier Current Signal at Different LeVel of Magnetizing CuRRENT AND AXIAL Rotor POSITION

\begin{tabular}{|c|c|c|c|c|c|c|c|c|c|}
\hline \multirow{2}{*}{$\mathbf{I}_{\text {sd }}$} & \multicolumn{9}{|c|}{ Rotor Axial Position - $x$} \\
\hline & $0 \mathrm{~mm}$ & $0.5 \mathrm{~mm}$ & $1 \mathrm{~mm}$ & $1.5 \mathrm{~mm}$ & $2 \mathrm{~mm}$ & $2.5 \mathrm{~mm}$ & $3 \mathrm{~mm}$ & $3.5 \mathrm{~mm}$ & $4 \mathrm{~mm}$ \\
\hline $4.8 \mathrm{~A}$ & 0.013436 & 0.01384 & 0.015281 & 0.016338 & 0.018251 & 0.019301 & 0.022991 & 0.026576 & 0.032322 \\
\hline $4.5 \mathrm{~A}$ & 0.011826 & 0.012139 & 0.013386 & 0.014058 & 0.015267 & 0.015638 & 0.018443 & 0.021493 & 0.025717 \\
\hline $4.0 \mathrm{~A}$ & 0.010252 & 0.010345 & 0.010961 & 0.011605 & 0.012217 & 0.012837 & 0.013457 & 0.015407 & 0.017626 \\
\hline $3.5 \mathrm{~A}$ & 0.009504 & 0.00955 & 0.009974 & 0.009993 & 0.010008 & 0.010099 & 0.010828 & 0.011916 & 0.012954 \\
\hline $3.0 \mathrm{~A}$ & 0.009092 & 0.009253 & 0.009413 & 0.009573 & 0.009733 & 0.009893 & 0.010054 & 0.010214 & 0.010374 \\
\hline $2.5 \mathrm{~A}$ & 0.00858 & 0.008597 & 0.008614 & 0.008631 & 0.008648 & 0.008665 & 0.008682 & 0.008699 & 0.008716 \\
\hline $2.0 \mathrm{~A}$ & 0.008067 & 0.008126 & 0.008184 & 0.008243 & 0.008302 & 0.00836 & 0.008419 & 0.008477 & 0.008536 \\
\hline $1.5 \mathrm{~A}$ & 0.007612 & 0.007705 & 0.007798 & 0.007891 & 0.007984 & 0.008077 & 0.00817 & 0.008263 & 0.008356 \\
\hline
\end{tabular}

\section{ACKNOWLEDGMENT}

The research leading to these results has received funding from the People Programme (Marie Curie Actions) of the European Union's Seventh Framework Programme (FP7/2007-2013) under REA grant agreement no 608322.

\section{REFERENCES}

[1] Safran Messier-Bugatti-Dowty, "Messier-Bugatti's "Green Taxiing ${ }^{\text {TM" }}$ solutions for Smarter Ground Operations," http://www.safranmbd.com/spip.php?article411\&lang=en.

[2] M. Galea, T. Hamiti, and C. Gerada, "Torque density improvements for high performance machines," in Electric Machines \& Drives Conference (IEMDC), 2013 IEEE International, 2013, pp. 1066-1073.

[3] T. Raminosoa, T. Hamiti, M. Galea, and C. Gerada, "Feasibility and electromagnetic design of direct drive wheel actuator for green taxiing," in Energy Conversion Congress and Exposition (ECCE), 2011 IEEE, 2011, pp. 2798-2804.

[4] M. Galea, S. Roggia, L. Papini, Z.Xu, C. Tighe, M. Hamiti, C. Gerada, S. Pickering, "Design aspects of a High Torque Density Machine for an Aerospace Traction Application," in Electrical Machines and Systems Conference (ICEMS), 2014 IEEE International, 2014, pp. 2773 - 2778.

[5] G. Munteanu, A. Binder, and S. Dewenter, "Five-Axis Magnetic Suspension with Two Conical Air Gap Bearingless PM Synchronous Half-Motors," in International Symposium on Power Electronics, Electrical Drives, Automation and Motion (SPEEDAM), 2012 IEEE International, 2012, pp. 1246 - 1251.
[6] M. M. Abdelfatah and F. P. Emad, "Conical Magnetic Bearings with Radial and Thrust Control," IEEE Transactions on Automatic Control, IEEE, 1992, pp. 1859-1862 vol. 37.

[7] F. Briz, M.W. Degner, A. Diez, R.D. Lorenz, "Measuring, modeling and decoupling of saturation-induced saliencies in carrier signal injectionbased sensorless AC drives," in Industry Applications Conference, 2000 IEEE, 2000, pp. 1842 - 1849 vol.3.

[8] N. Teske, G.M. Asher, M. Sumner, K.J Bradley, "Analysis and suppression of high-frequency inverter modulation in sensorless position-controlled induction machine drives," IEEE Transactions on Industry Applications, 2003 IEEE, , pp. 10-18 vol.39.

[9] S. Damkhi, M.S.N. Said, N.N. Said, "Slotting effects and high frequency signal injection for induction machine rotor speed estimation," in International Conference and Exposition on Electrical and Power Engineering (EPE), 2012 IEEE, pp. 401 - 408.

[10] M.W. Degner, R.D. Lorenz, " Position estimation in induction machines utilizing rotor bar slot harmonics and carrier-frequency signal injection," IEEE Transactions on Industry Applications, IEEE, 2000, pp. 736 - 742 vol.36.

[11] Jansen, P.L., R.D. Lorenz,"Trasducerless field Orientation Concept Employing Saturation-Induced Saliencies inInduction Machines, " Industry Applications Conference, IEEE, 1995,pp. 174 - 181 vol.1.

[12] N. Teske, G.M. Asher, M. Sumner, K.J. Bradley, "Suppression of Saturation Saliency Effects for the Sensorless Position Control of Induction Motor Drives Under Loaded Condition," Transactions on Industrial Electronics, 2000 IEEE, 2000, pp. 1142 - 1150 vol. 47.

[13] V. K. Ingle, J. G. Proakis, Digital Signal Processing using Matlab Third Edition, Cengage Learning, 2011. 